2

3

$\mathrm{a}^{*}$ Bernd Kastenholz, ${ }^{\mathrm{b}}$ Basil Horst, and ${ }^{\mathrm{c}}$ Jürgen Horst

4

5

$\mathrm{BK}, \mathrm{BH}$ and $\mathrm{JH}$ equally contributed to this paper.

6

7

8

9

10

11

12

13

14

15

16

17

18

19

20

21

22

23

24

25 


\section{Institute of origin:}

2 a IBG-2: Plant Sciences, Research Centre Juelich, 52425 Juelich, Germany

$3 \quad{ }^{\mathrm{b} C o l u m b i a}$ University, Department of Dermatology, New York, NY 10032, USA

4 ' Westfälische Wilhelms-Universität Münster, Institut für Humangenetik, 48149 Münster,

5 Germany

6

7 Corresponding author:

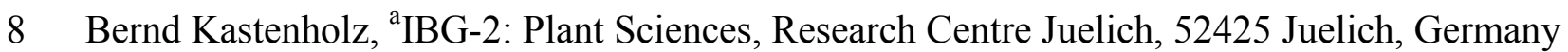

$9 \quad$ Tel.: +49 (0) 2461614825 ;

10 Fax: $+49(0) 2461612492$;

11 E-mail: b.kastenholz@fz-juelich.de

12

13 Running title:

14 Phytopharmaceuticals for restoring biometal homeostasis in human cells

16 Key words:

17 Alzheimer's disease (AD), recombinant plant-made pharmaceuticals (PMPs), CCS, Ginkgo

18 biloba, SOD, A $\beta$, molecular chaperones

\section{Abbreviations:}

21 CCS: copper chaperone for superoxide dismutase

22 SOD-1: $\mathrm{Cu}, \mathrm{Zn}$-superoxide dismutase

23 A $\beta$ : amyloid $\beta$

24 AD: Alzheimer's disease

25 ROS: reactive oxygen species

26 PMPs: plant-made pharmaceuticals 
1 Abstract:

2 Therapeutic recombinant plant-made copper chaperone for superoxide dismutase (CCS) 3 derived from Ginkgo biloba leaves may establish and maintain physiologic $\mathrm{Cu}$ levels through 4 restoration and modulation of biometal metabolism in organ systems of younger Alzheimer 5 patients ( $>50$ years). Medications developed from plant-made copper chaperone proteins may 6 delay progression during early disease stages or even be a basis for a possible causal treatment 7 of preclinical stages of Alzheimer's disease by restoring cellular function of the CCS-SOD-1 8 mechanism and by preventing formation of $A \beta$ plaques, a major putative factor involved in 9 Alzheimer's disease pathogenesis.

10

11

12

13

14

15

16

17

18

19

20

21

22

23

24

25

26 


\section{INTRODUCTION}

2 The dysregulation of biometal $(\mathrm{Cu}, \mathrm{Zn}, \mathrm{Fe})$ homeostasis and oxidative stress in brain cells have been found to impact on accumulation of amyloid $\beta(A \beta)$, a major putative factor involved in early Alzheimer's disease (AD) pathogenesis [1]. The regulation of metal ion homeostasis in the cytoplasm is strongly influenced by copper chaperone for superoxide dismutase (CCS) and $\mathrm{Cu}, \mathrm{Zn}$-superoxide dismutase (SOD-1) [2]. The dynamic interplay of properly folded CCS and SOD-1 guarantees that free $\mathrm{Cu}$ and $\mathrm{Zn}$ ions are being complexed by these metal proteins and do not catalyze oxidation processes of proteins, lipids, DNA, and other molecules in the cells [2]. When these physiological complexation mechanisms do not function properly, oxidative stress and dys-homeostasis of $\mathrm{Cu}$ and $\mathrm{Zn}$ metabolism may give rise to misfolding, accumulation and aggregation of amyloid $\beta$ peptides [2]. The outcome of these pathological processes may lead to incurable chronically progressive neurodegenerative diseases such as Alzheimer's disease [1,2]. Several therapeutic strategies and nearly all medications used or suggested as $A \beta$ inhibitors, including metal-chelating agents or radical scavengers, at present time, aim at the treatment of AD symptoms only and may either be toxic, lack specificity or have unknown mechanisms of action in vivo $[2,3]$.

The aim of this article is to give a short review on the interaction of metal ions with novel and early herbal compounds derived from Ginkgo biloba, and their possible role in the treatment of early Alzheimer's disease. Though Ginkgo biloba leaf extracts are generally administered to treat dementia syndroms in older AD patients ( $>65$ years old), no data is available on possible effects of recombinant plant-made copper chaperones from Ginkgo biloba in younger AD patients ( $>50$ years old) with preclinical stages of disease.

\section{INTERACTION OF HERBAL DRUGS WITH METAL IONS}

The medical community, including the pharmaceutical industry, as well as AD patients, have become aware of the well-known antioxidant effects of ancient herbal medications, namely Ginkgo biloba leaf extracts [4]. A complex mixture of flavonoids and terpenoids are thought to represent the main bioactive compounds of these plant extracts [5]. For example, the flavonol quercetin, a major compound of Ginkgo extract, was found to interact with $\mathrm{Cu}^{2+}$ and $\mathrm{Fe}^{3+}$ [6]. However, an ideal therapeutic drug to dissolve $\mathrm{A} \beta$ peptides would involve a compound selective for $\mathrm{Cu}^{+}, \mathrm{Zn}^{2+}$ and $\mathrm{Fe}^{3+}$ [7]. A meta-analysis by Birks and Evans revealed that commercially available medicinal plant extracts (EGb $761^{\circledR}$ Ginkgo biloba leaf extract) have no consistent pattern of any clinical benefit associated with Ginkgo biloba for people 
1 with dementia or cognitive impairment [4]. Furthermore, the results from the study of $\mathrm{He}$ and colleagues suggested that high doses of herbal remedies can even be toxic to cells [8]. Thus, Ginkgo biloba extracts may induce unwanted side-effects and may also lack specificity as to the binding of $\mathrm{Cu}^{+}$and $\mathrm{Zn}^{2+}$ in the cytoplasm. Likely, these extracts neither reduce metalbased oxidative stress efficiently nor contribute to the homeostatic control of biometals in human cells, though in vitro studies have shown that gingkolides may protect against the synapse damage and cognitive loss seen during the early stages of $\mathrm{AD}$ [5]. However, medicinal plants may contain other, more efficient bioactive molecules apart from the wellknown flavonoids and terpenoids, namely metallochaperones [9].

\section{EFFICACY OF PLANT-MADE COPPER CHAPERONES}

As a basis for a new drug development involving metal-chelating agents, the following facts may be important. Endogenous biomolecules such as $\mathrm{Cu}, \mathrm{Zn}$-superoxide dismutase, are one of the major means by which cells counteract the deleterious effects of reactive oxygen species (ROS). For proper functioning SOD-1 has to be activated by the metallochaperone protein, copper chaperone for SOD [9-12]. Copper ions are required for enzymatic activity whereas the zinc ion helps to stabilize the enzyme [10]. Studies by Choi and colleagues suggest that recombinant human CCS molecules produced in bacteria provide a potential strategy for therapeutic delivery of these compounds in various human diseases related to ROS and SOD [10]. Drugs derived from recombinant proteins potentially have greater efficacy and fewer side-effects than small organic molecules (e.g., $\mathrm{Cu}$ orotate, quercetin), because their action can be more precisely targeted towards the $A \beta$ plaque formation as a major putative factor in the pathogenesis of Alzheimer's disease rather than the treatment of AD symptoms [11]. The dysfunction of the CCS-SOD-1 interplay may be one specific but major mechanism in the pathogenesis of Alzheimer's disease. It is a well-known fact that biometals (Fe, $\mathrm{Zn}, \mathrm{Cu}$ ) are accumulated in the brain with normal ageing [13]. Important factors affecting the balance between metal ion accumulation and deficiency are, for example, environmental exposure, ageing or drug interaction [14]. The inability of the human organism to maintain the metal ion homeostasis due to improperly folded CCS and SOD molecules in brain cells is suggested as causal for preclinical stages, development and progression of $\mathrm{AD}$ and other neurodegenerative diseases $[9,11,15]$.

For the restoration and modulation of metal ion homeostasis in the treatment of $\mathrm{AD}$, we have proposed a novel class of pharmacologically active plant ingredients as antioxidants: copper 
1 chaperone for superoxide dismutase derived from medicinal plants (e.g., Ginkgo biloba) 2 [9,11]. In molecular farming approaches transgenic plants (e.g., tobacco) may serve as an 3 efficient production platform for medications in regard to protein yield, quality and stability 4 [16]. Recombinant CCS proteins produced in transgenic plants may cross the blood-brain barrier and are relatively free from side-effects [9,11]. Being properly-folded, plant-made CCS may have the ability to bind and deliver $\mathrm{Cu}^{+} / \mathrm{Cu}^{2+}, \mathrm{Zn}^{2+}$ and $\mathrm{Fe}^{3+}$ ions and to normalize the SOD-1 activity via specific protein-protein interactions in the central nervous system and peripherally $[9,11]$.

In contrast to plant-made pharmaceuticals (PMPs), organisms like yeast, mammals or bacteria used for molecular farming approaches, express properly folded as well as improperly folded recombinant therapeutic proteins. These medications may lack stability and pharmacological efficiency in protein-misfolding diseases, e.g., Alzheimer's disease [16]. Furthermore, PMPs may have several advantages in terms of cost scalability or safety issues compared to the other genetically modified organisms [16].

Because of their specific biochemical behavior, plant-made CCS from medicinal plants may be efficient in the treatment of patients with preclinical stages of AD. The endogenous levels of this essential copper protein may be important, since a mild copper deficiency has been described in AD patients [17]. Furthermore, the expression level of CCS has been found to reflect the $\mathrm{Cu}$ status of patients and thus, may serve as a marker for in vivo copper levels [18]. The metal levels in patients with Alzheimer's disease compared with healthy individuals are important parameters for developing drugs that may restore the intracellular metal ion metabolism [14]. It is anticipated that plant-made CCS may establish physiologic copper levels through restoration and modulation of biometal metabolism in diseased organ systems of $\mathrm{AD}$ patients [9,11]. Yet, to our knowledge, no systematic studies have addressed the possible role of plant-made CCS in copper homeostasis.

The effects of recombinant CCS and a possible role in the treatment of $\mathrm{AD}$ can be characterized as follows: unfolded SOD-1 in diseased blood involving denatured proteins and peptides [19], may be activated by copper ion incorporation via specific CCS-SOD interaction $[9,11]$. Copper-demetallated CCS and $\mathrm{Cu}$ cofactor-containing CCS complexes may pass through the blood-brain barrier and activate unfolded SOD-1 by $\mathrm{Cu}$ ion transfer or bind free 
1 an important possible factor in the complex pathogenesis of $\mathrm{AD}$ [20] restoring homeostasis of

$2 \mathrm{Cu}$ metabolism may positively affect early disease stages of $\mathrm{AD}$ patients by balancing oxidative and anti-oxidative processes and by reducing protein-misfolding processes leading to formation of $\mathrm{A} \beta$ plaque deposits $[9,11,20]$. The interactions of plant-derived CCS medications with unfolded human SOD-1 and free metal ions $(\mathrm{Cu}, \mathrm{Zn}, \mathrm{Fe})$ may trigger a cascade of other biochemical reactions, such as the degradation of $\mathrm{A} \beta$ plaque deposits by molecular chaperones and the ubiquitin proteasome system [21].

Further studies to determine the dose, bioavailability and mechanisms of action of plant-made pharmaceuticals seem warranted in younger AD patients ( $>50$ years) as previously proposed $[9,11]$.

\section{CONCLUSION}

For the restoration and modulation of metal ion homeostasis and for balancing intracellular pro-oxidative and antioxidative processes in the treatment of Alzheimer's disease, plant-made copper chaperone for superoxide dismutase (CCS) proteins potentially have greater efficacy and fewer side-effects compared to small organic molecules (e.g., quercetin) from medicinal plant extracts or therapeutic recombinant proteins produced in bacteria, fungi or mammals. Possibly suitable for use in younger AD patients ( $>50$ years), CCS proteins derived from medicinal plants may be targeted more towards prevention of $\mathrm{A} \beta$ plaque formation as a major putative factor involved in $\mathrm{AD}$ pathogenesis, rather than the treatment of $\mathrm{AD}$ symptoms in older patients (> 65 years). We suggest that recombinant plant-made CCS derived from Ginkgo biloba leaves might be promising in the treatment of patients suffering from preclinical symptoms of Alzheimer's disease.

\section{REFERENCES}

1. Wesson, D.W.; Nixon, R.A.; Levy, E.; Wilson, D.A. Mechanisms of Neural and 8 Behavioral Dysfunction in Alzheimer's Disease. Mol. Neurobiol., 2011, in press.

9 2. Crichton, R.R.; Ward, R.J. Metal-Based Neurodegeneration: From Molecular Mechanisms to Therapeutic Strategies, 1st ed.; John Wiley \& Sons Ltd.: Chichester, 1 England, 2006.

3. Estrada, L.D.; Yowtak, J.; Soto, C. Protein misfolding disorders and rational design of 3 antimisfolding agents. Methods Mol. Biol., 2006, 340, 277-293. 
14 Birks, J.; Evans, J.G. Ginkgo biloba for cognitive impairment and dementia. Cochrane Database Syst. Rev., 2009, DOI:10.1002/14651858.CD003120.pub3.

5. Bate, C.; Tayebi, M.; Williams, A. Ginkgolides protect against amyloid-beta (1-42)mediated synapse damage in vitro. Mol. Neurodegen., 2008, 3, Article No.: 1.

6. Kuo, S.-M.; Leavitt, P.S.; Lin, C.-P. Dietary flavonoids interact with trace metals and affect metallothionein level in human intestinal cells. Biol. Trace Elem. Res., 1998, 62(3), 135-153.

7. Cuajungco, M.P.; Faget, K.Y.; Huang, X.; Tanzi, R.E.; Bush A.I. Metal chelation as a potential therapy for Alzheimer's disease. Ann. N.Y. Acad. Sci., 2000, 920, 292-304.

8. He, J.; Lin, J.; Li, J.; Zhang, J.-H.; Sun, X.-M.; Zeng, C.-M. Dual effects of Ginkgo biloba leaf extract on human red blood cells. Basic Clin. Pharmacol. Toxicol., 2009, 104(2), 138-144.

9. Kastenholz, B.; Garfin, D.E. Medicinal plants: a natural chaperones source for treating neurological disorders. Protein Pept. Lett., 2009, 16(2), 116-120.

10. Choi, S.H.; Kim, D.W.; Kim, S.Y.; An, J.J.; Lee, S.H.; Choi, H.S.; Sohn, E.J.; Hwang, S.-I.; Won, M.H.; Kang, T.-C.; Kwon, H.J.; Kang, J.H.; Cho, S.-W.; Park, J.; Eum, W.S.; Choi, S.Y. Transduced human copper chaperone for Cu,Zn-SOD (PEP-1-CCS) protects against neuronal cell death. Mol. Cells, 2005, 20(3), 401-408.

11. Kastenholz, B.; Garfin, D.E.; Horst, J.; Nagel, K.A. Plant metal chaperones: a novel perspective in dementia therapy. Amyloid J. Prot. Fold. Disord., 2009, 16(2), 81-83.

12. Sekhon, B.S. Metallochaperones - an overview. Curr. Chem. Biol., 2010, 4(2), 173186.

13. Smith, D.G.; Cappai, R.; Barnham, K.J. The redox chemistry of the Alzheimer's disease amyloid beta peptide. Biochim. Biophys. Acta - Biomembr., 2007, 1768(8), 1976-1990.

14. Zatta, P.; Drago, D.; Bolognin, S.; Sensi S.L. Alzheimer's disease, metal ions and metal homeostatic therapy. Trends Pharmacol. Sci., 2009, 30(7), 346-355.

15. Bolognin, S.; Messori, L.; Zatta, P. Metal ion physiopathology in neurodegenerative disorders. Neuromol. Med., 2009, 11(4), 223-238.

16. Nagel, K.A.; Kastenholz, B.; Gilmer, F.; Schurr, U.; Walter, A. Novel detection system for plant protein production of pharmaceuticals and impact on conformational diseases. Protein Pept. Lett., 2010, 17(6), 723-731.

17. Pajonk, F.-G.; Kessler, H.; Supprian, T.; Hamzei, P.; Bach, D.; Schweickhardt, J.;

Herrmann, W.; Obeid, R.; Simons, A.; Falkai, P.; Multhaup, G.; Bayer, T.A. Cognitive 
decline correlates with low plasma concentrations of copper in patients with mild to moderate Alzheimer's disease. J. Alz. Dis., 2005, 8(1), 23-27.

18. Wang, T.; Guo, Z.J. Copper in medicine: homeostasis, chelation therapy and antitumor drug design. Curr. Med. Chem., 2006, 13(5), 525-537.

19. Kang, M.N.; Park, W.; Kang, S.M.; Lim, K.; Lee, B.; Kim, G.J.; Kim, S.Y.; Yang, Y.S.; Na, H.R.; Youn, Y.C.; An, S.S.A.; Ju, Y.R.; Kim, S. Presence of amyloid-beta (A $\beta$ ) oligomers in blood from patients with Alzheimers disease. Prion, 2010, 4(3), 224-224.

20. Lin, C.-J., Huang, H.-C., Jiang, Z.-F. Cu(II) interaction with amyloid- $\beta$ peptide: a review of neuroactive mechanisms in AD brains. Brain Res. Bull., 2010, 82(5-6), $235-$ 242.

21. Luo, G.R.; Le, W.D. Collective roles of molecular chaperones in protein degradation pathways associated with neurodegenerative diseases. Curr. Pharm. Biotechnol., 2010, 11(2), 180-187. 